https://doi.org/10.22319/rmcp.v11s2.4693

Artículo

\title{
Áreas con aptitud ambiental para [Bouteloua curtipendula (Michx.) Torr.] en México por efecto del cambio climático
}

José Ángel Martínez Sifuentes a

Noé Durán Puga ${ }^{a^{*}}$

José Ariel Ruiz Corral ${ }^{\text {a }}$

Diego Raymundo González Eguiarte ${ }^{a}$

Salvador Mena Munguía ${ }^{a}$

${ }^{a}$ Universidad de Guadalajara. Centro Universitario de Ciencias Biológicas y Agropecuarias. Camino Ramón Padilla Sánchez No. 2100 Nextipac, 44600, Zapopan, Jalisco, México.

*Autor de correspondencia: noe.duran@ cucba.udg.mx

\section{Resumen:}

Las áreas de pastizal están expuestas a los efectos del cambio climático que se presentarán en las siguientes décadas, lo que provocará cambios en las comunidades vegetales, modificando a su vez los servicios y productos. La influencia del clima como determinante de la productividad primaria en los ecosistemas ha llevado a la realización de estudios para evaluar el impacto del cambio climático en comunidades vegetales mediante el uso de modelos de simulación. Las especies del género Bouteloua están entre las más importantes en los pastizales de México, por su calidad forrajera y sus características ecológicas; entre ellas sobresale el zacate Banderita [Bouteloua curtipendula (Michx.) Torr.]. El objetivo del presente estudio fue delimitar las áreas con aptitud ambiental de $B$. curtipendula por efecto del cambio climático en México. El análisis del clima futuro se hizo con los modelos de circulación general (MCG) HadGEM-ES y GFDL-CM3, con los RCP4.5 y RCP8.5, para los periodos 2041-2060 y 2061-2080; para modelar los nichos potenciales de distribución se usaron datos de 407 sitios georreferenciados de colecta y 29 variables ambientales; mediante el modelo MaxEnt ambos MCG pronostican que el área con aptitud ambiental para $B$. 
curtipendula tendrá una disminución inicial entre 3.1 a $14.4 \%$, aunque después se recuperará e incluso aumentará hasta un $1.4 \%$. Las variables ambientales que más contribuyeron a explicar la distribución potencial de la especie fueron la oscilación térmica anual, la precipitación en el periodo mayo a octubre y el índice de humedad de diciembre a febrero.

Palabras clave: Bouteloua curtipendula, Aptitud ambiental, Descriptores, Nicho ecológico, Maxent, Modelos de circulación general, Cambio climático.

Recibido: 12/11/2017

Aceptado: 12/07/2018

\section{Introducción}

La superficie de pastoreo en México es superior al $45 \%$ del territorio nacional, con una mayor proporción en la región norte del país donde llega hasta el $70 \%{ }^{(1)}$, sin embargo, estas áreas de pastizal están expuestas a los efectos del cambio en las condiciones del clima que se presentarán en las siguientes décadas de acuerdo con diversos estudios realizados, lo que provocará cambios en las comunidades vegetales, así como los servicios y productos que proveen $^{(2,3)}$. La influencia del clima como determinante de la productividad primaria de los ecosistemas ha llevado a la realización de estudios con el fin de evaluar el impacto del cambio climático en las comunidades vegetales mediante el uso de modelos de simulación. Algunas investigaciones realizadas en México han mostrado que la temperatura ambiental se incrementará entre 1.8 y $4.5^{\circ} \mathrm{C}$ durante el periodo 2040-2100, en tanto que la precipitación pluvial disminuirá del 2 al $12 \%{ }^{(4,5,6)}$.

Las especies del género Bouteloua son de las más importantes en los pastizales de México, por su calidad forrajera y sus características ecológicas, entre las cuales sobresale el zacate Banderita o Banderilla [Bouteloua curtipendula (Michx.) Torr.]; esta especie ha sido incluida en algunos programas de mejoramiento de pastizales, por sus características forrajeras sobresalientes e incluso se han liberado variedades mejoradas en México ${ }^{(7,8)}$ y en Estados Unidos $^{(9)}$. Además, B. curtipendula tiene una amplia variabilidad del polimorfismo que podría darle ventaja de adaptación ante los efectos del cambio climático ${ }^{(10)}$.

En algunos estudios se ha reportado que la estructura de las comunidades vegetales depende en gran medida de las condiciones climáticas, entre las cuales sobresalen la precipitación y la temperatura como factores determinantes ${ }^{(11,12,13)}$; así mismo, se ha encontrado que la sequía crónica disminuye fuertemente la cobertura de las gramíneas al mismo tiempo que se incrementa la cobertura de especies arbustivas, en algunas áreas del desierto chihuahuense ${ }^{(13)}$. 
Otros resultados ${ }^{(14)}$ muestran que hay poca evidencia de que los cambios en la precipitación influirán en los efectos competitivos individuales de las plantas, en un área de pastizal con dominancia de Bouteloua curtipendula, Bouteloua hirsuta y Schizachyrium scoparium, y que son las interacciones intraespecíficas e interespecíficas las que pueden modificar la colonización de espacios con características agroecológicas poco adecuadas. El objetivo de la presente investigación fue analizar las áreas con aptitud ambiental de B. curtipendula por efecto del cambio climático en México; utilizando dos modelos de circulación general (MCG) bajo las vías representativas de concentración de gases efecto invernadero (RCP4.5 y RCP8.5), para los periodos 2041-2060 y 2061-2080.

\section{Material y métodos}

\section{Bases de datos y sistemas de información ambiental}

La investigación se basó en el análisis de los datos del clima de referencia y la modelación de los climas futuros, datos obtenidos del portal de Global Climate Data de WorldClim.org. Para la climatología de referencia se utilizaron los datos del periodo 1950-2000 y para el clima futuro los correspondientes a los periodos 2041-2060 y 2061-2080, referidos más adelante como periodos 2050 y 2070, respectivamente, con una resolución espacial de 30 segundos de $\operatorname{arco}^{(15)}$.

Se utilizaron los MCG HadGEM-ES y GFDL-CM3; el primero se seleccionó porque incluye como variable los tipos de vegetación, incluido el pastizal nativo como parte de la cubierta vegetal del planeta $^{(16)}$; el segundo debido a que la versión CM3, además de incluir temas emergentes del cambio climático, tiene una resolución espacial mejorada y pone atención especial en la simulación de la precipitación en áreas tropicales ${ }^{(17)}$. En la simulación se usaron las vías representativas de concentración de gases efecto invernadero ${ }^{(18)}(\mathrm{RCP}$, por sus siglas en inglés) de 4.5 y 8.5 para analizar un escenario bajo y uno alto; no se usó el RCP2.6 debido a que las tendencias muestran dificultad para lograr este escenario ${ }^{(19)}$. Los datos del clima se procesaron con el software ArcGIS; después se generaron mapas con las variables climáticas y bioclimáticas con el software Idrisi Selva, imágenes que se utilizaron para analizar las áreas con aptitud ambiental para B. curtipendula con el modelo Maximum Entropy Species Distribution Modeling (MaxEnt).

\section{Distribución potencial de $B$. curtipendula}

Para correr el modelo Maxent se hicieron los siguientes ajustes: uso del $25 \%$ de los datos para prueba, 10 replicaciones con la prueba de validación cruzada y un máximo de 2,000 iteraciones. Se utilizaron 410 datos georreferenciados obtenidos de cuatro fuentes: a) colecta directa, b) colecta de otros investigadores, c) datos consignados en ejemplares del herbario 
del Instituto de Biología del Centro Universitario de Ciencias Biológicas y Agropecuarias de la Universidad de Guadalajara, y d) datos de colectas publicados en el sitio web de Global Biodiversity Information Facility ${ }^{(20)}$.

Las variables ambientales utilizadas fueron: precipitación anual, precipitación mayo-octubre, precipitación noviembre-abril, precipitación diciembre-febrero, precipitación en el mes más húmedo, precipitación en el mes más seco, temperatura máxima anual, temperatura máxima mayo-octubre, temperatura máxima noviembre-abril, temperatura media anual, temperatura media mayo-octubre, temperatura media noviembre-abril, temperatura media del mes más cálido, temperatura media del mes más frío, temperatura mínima anual, temperatura mínima mayo-octubre, temperatura mínima noviembre-abril, fotoperiodo mayo-octubre, fotoperiodo noviembre-abril, índice de humedad anual, índice de humedad mayo-octubre (estimado como el cociente de la precipitación entre evapotranspiración), índice de humedad noviembre-abril, índice de humedad diciembre-febrero, oscilación térmica anual, oscilación térmica mayooctubre, oscilación térmica noviembre-abril, oscilación térmica diciembre-febrero y textura del suelo.

\section{Áreas con probabilidad de aptitud ambiental}

El modelo de predicción de áreas con aptitud ambiental para B. curtipendula obtenido con Maxent fue utilizado con el sistema Idrisi $17.0^{(21)}$ con el que se generó un mapa con los valores umbrales correspondientes al percentil $10^{(22)}$. Para el cálculo de la superficie con aptitud ambiental de la especie, no se consideraron las áreas ocupadas por los cuerpos de agua y centros urbanos; estas capas temáticas se obtuvieron mediante la carta del uso del suelo y vegetación ${ }^{(23)}$.

\section{Ajuste del modelo}

El modelo de nicho ecológico empleado por Maxent predice la tasa de ocurrencia relativa (Receiver operating characteristic, ROC) de la especie, como una función de los predictores ambientales en cada localidad ${ }^{(24)}$, representada por cada celda de la malla de aproximadamente 900 x 900 m en la escala geográfica de 30 seg de arco; a su vez, el área bajo la curva (Area Under Curve, AUC) puede interpretarse como el ajuste del modelo, en el

que un valor de 1.0 sería un clasificador perfecto y uno aleatorio tendría un valor de $0.5^{(25)}$, por lo que los valores cercanos a 1.0 muestran mayor ajuste del modelo a los datos. 


\section{Resultados y discusión}

\section{Análisis del nicho potencial de distribución}

Los valores promedio de AUC obtenidos para el clima de referencia y para los escenarios de cambio climático fueron mayores a 0.933 en todos los casos (Cuadro 1), por lo que se considera que los datos obtenidos tienen un alto grado de confiabilidad al evaluar la aptitud ambiental para $B$. curtipendula ${ }^{(24,25)}$. Resultados que concuerdan parcialmente en una investigación realizada en pastizales de Estados Unidos ${ }^{(26)}$ en donde el análisis para $B$. curtipendula con 1,251 datos de muestreo, refleja un valor del AUC de 0.946; el valor más alto de la AUC de este estudio puede deberse a un mayor número de referencias geográficas utilizadas. Por otra parte, en las 10 réplicas del modelo utilizadas en esta investigación se encontró que el valor más bajo de AUC fue de 0.915 y el más alto de 0.976, la desviación estándar fue $<0.013$ en todos los casos, por lo que los resultados se consideran confiables. Ambos MCG arrojaron datos similares en la predicción de áreas con aptitud potencial, por lo que en investigaciones posteriores podría utilizar cualquiera de ellos.

Cuadro 1: Valores promedio del área bajo la curva ROC, obtenidos en 10 replicaciones, y desviación estándar promedio, en el análisis de distribución potencial de Boutelua

\section{curtipendula en México}

\begin{tabular}{lcccc}
\hline \multicolumn{1}{c}{ MCG } & $\begin{array}{c}\text { AUC } \\
\text { promedio }\end{array}$ & $\begin{array}{c}\text { AUC más } \\
\text { alto }\end{array}$ & $\begin{array}{c}\text { AUC más } \\
\text { bajo }\end{array}$ & $\begin{array}{c}\text { Desviación } \\
\text { estándar }\end{array}$ \\
\hline Clima de referencia & 0.934 & 0.955 & 0.920 & 0.011 \\
GFDL-CM3 RCP4.5 2050 & 0.933 & 0.953 & 0.915 & 0.011 \\
GFDL-CM3 RCP4.5 2070 & 0.955 & 0.966 & 0.928 & 0.013 \\
GFDL-CM3 RCP8.5 2050 & 0.956 & 0.969 & 0.932 & 0.011 \\
GFDL-CM3 RCP8.5 2070 & 0.935 & 0.953 & 0.920 & 0.011 \\
HadGEM-ES RCP4.5 2050 & 0.957 & 0.975 & 0.932 & 0.012 \\
HadGEM-ES RCP4.5 2070 & 0.937 & 0.960 & 0.923 & 0.012 \\
HadGEM-ES RCP8.5 2050 & 0.956 & 0.976 & 0.928 & 0.013 \\
HadGEM-ES RCP8.5 2070 & 0.934 & 0.951 & 0.920 & 0.010 \\
\hline
\end{tabular}

MCG= modelos de circulación general; $\mathrm{AUC}=$ área bajo la curva. 


\section{Cambios en la temperatura media anual y la precipitación acumulada}

En el análisis referente a la temperatura media anual, los dos MCG utilizados en esta investigación pronostican incrementos promedio de 2.8 y $3.4{ }^{\circ} \mathrm{C}$ para el periodo 2050 y de 3.4 y $5.0^{\circ} \mathrm{C}$ para el periodo 2070 con el RCP4.5 y RCP8.5, respectivamente. Con respecto a la precipitación acumulada anual los dos modelos predicen un decremento en la climatología 2050 de 3.12 y $6.5 \%$ y de 7 y $14.4 \%$ para la climatología 2070 . Es importante señalar que los cambios en la temperatura y la precipitación serán diferentes en cada área geográfica, con tendencia general a que sea más acentuado el cambio en las zonas áridas y semiáridas que en las zonas templadas y tropicales de México.

\section{Áreas con aptitud ambiental de B. curtipendula}

En la Figura 1 se observa que la especie se distribuye de manera natural en una extensión de $548,719 \mathrm{~km}^{2}$ (Cuadro 2), localizada en el centro y norte de México; desde el sureste del estado de Chihuahua hasta la parte norte de Michoacán y Guerrero, en las áreas de pastizal nativo ubicadas en la altiplanicie mexicana y el eje volcánico transversal. Es notoria la baja presencia de la especie y poca superficie con aptitud ambiental en las áreas bajas y planicies costeras en los litorales del Océano Pacífico, Golfo de México y Mar Caribe.

Figura 1: Área actual con aptitud ambiental de B. curtipendula en México

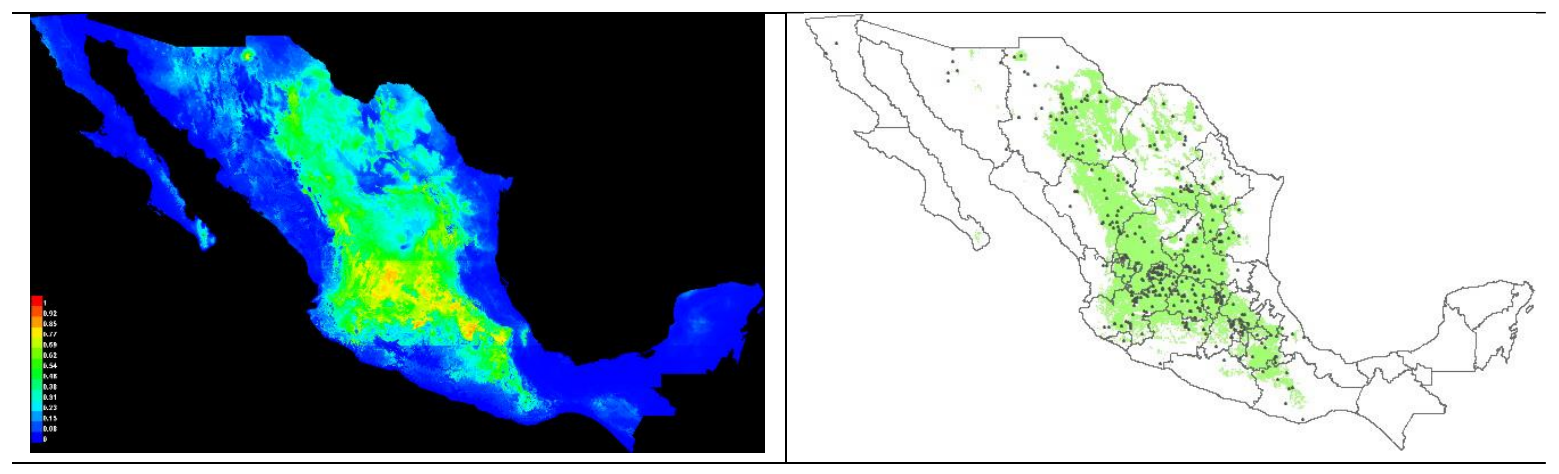

Lado izquierdo: mapa del modelo Maxent, color rojo con mayor probabilidad de ocurrencia; color azul áreas con menor probabilidad. Lado derecho: Mapa basado en el anterior, con la superficie de aptitud ambiental limitada en el decil 10 del valor de probabilidad; los puntos indican los sitios de colecta de la especie.

Los resultados obtenidos con el algoritmo Maxent son influidos por los datos ambientales utilizados $^{(27,28)}$; en la Figura 1 (lado derecho) se muestra el área homogénea con mayor aptitud ambiental con el clima de referencia y en la Figura 2 se pueden observar las áreas con aptitud ambiental para los periodos futuros y los RCP analizados. 
Cuadro 2: Superficie con aptitud ambiental actual de Bouteloua curtipendula en México y variación con los modelos de circulación general (MCG)

\begin{tabular}{ccc}
\hline Clima/MCG & $\begin{array}{c}\text { Superficie con } \\
\text { aptitud ambiental } \\
\left(\mathbf{k m}^{2}\right)\end{array}$ & $\begin{array}{c}\text { \% en relación con la } \\
\text { climatología de referencia }\end{array}$ \\
\hline Referencia & 548,719 & 100.0 \\
GFDL-CM3 RCP4.5 2050 & 509,152 & 92.8 \\
GFDL-CM3 RCP4.5 2070 & 505,516 & 92.1 \\
GFDL-CM3 RCP8.5 2050 & 521,876 & 95.1 \\
GFDL-CM3 RCP8.5 2070 & 557,293 & 101.6 \\
HadGEM-ES RCP4.5 2050 & 506032 & 92.2 \\
HadGEM-ES RCP4.5 2070 & 520457 & 94.8 \\
HadGEM-ES RCP8.5 2050 & 528419 & 96.3 \\
HadGEM-ES RCP8.5 2070 & 552,799 & 100.7 \\
\hline
\end{tabular}

Los MCG convergen en la predicción de un ligero decremento de la superficie con aptitud ambiental para B. curtipendula para las dos futuras climatologías (con excepción en el RCP8.5 en el 2070); la disminución de la superficie registrada se localiza principalmente en la zona oriental del estado de Chihuahua, el norte de Durango, el noreste de Coahuila y en pequeñas áreas distribuidas en el centro y sur de la república mexicana (Figura 2). En referencia al RCP8.5 periodo 2070 se pronostica un aumento promedio de $1.6 \%$ de la superficie (Cuadro 2), localizada principalmente en el noreste de Chihuahua y centro-norte de Coahuila, además de pequeñas áreas dispersas en otras zonas aledañas al área con aptitud ambiental actual. Este fenómeno puede estar influenciado por el tipo de metabolismo de la especie y en este caso la fisiología de $B$. curtipendula es del tipo $C 4^{(26)}$, por lo cual es más eficiente en el uso de agua y temperaturas altas ${ }^{(3,29)}$.

\section{Descriptores ecológicos de la distribución geográfica de B. curtipendula}

La oscilación térmica y la precipitación fueron los descriptores ecológicos que más contribuyeron en la distribución potencial del pasto B. curtipendula en México (Cuadro 3) en todos los escenarios ambientales. Otras investigaciones también han mostrado la influencia que tienen la precipitación y la temperatura ambiental en esta especie ${ }^{(3,13)}$. Al analizar la oscilación térmica por separado de las otras variables (Figura 3) se observó un fuerte incremento en la probabilidad de ocurrencia de B. curtipendula entre 14 y 20 grados de diferencia entre las temperaturas máxima y mínima (la oscilación térmica). 
Figura 2: Áreas con aptitud ambiental para B. curtipendula y cambios estimados para los periodos 2041-2060 y 2061-2080, con RCP4.5 y RCP8.5, en relación con el clima de referencia

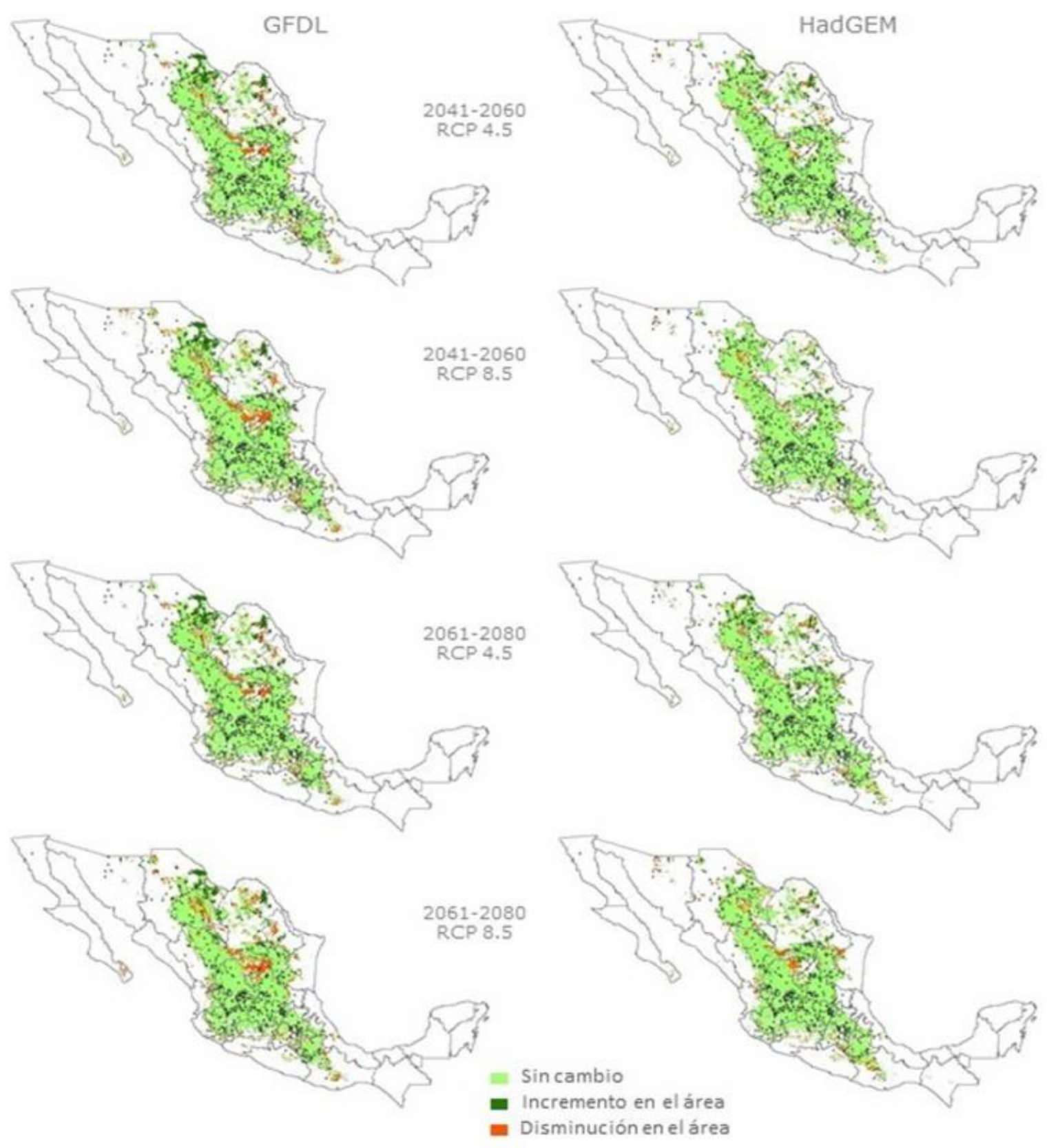


Cuadro 3: Contribución relativa (\%) de las variables ambientales que tienen mayor influencia en la aptitud ambiental para Bouteloua curtipendula en México

\begin{tabular}{llllllllll}
\hline & & \multicolumn{4}{c}{ RCP 4.5 } & \multicolumn{5}{c}{ RCP 8.5 } \\
\cline { 3 - 11 } Variable ambiental & referencia & $\mathbf{2 0 5 0}$ & $\mathbf{2 0 5 0}$ & $\mathbf{2 0 7 0}$ & $\mathbf{2 0 7 0}$ & $\mathbf{2 0 5 0}$ & $\mathbf{2 0 5 0}$ & $\mathbf{2 0 7 0}$ & $\mathbf{2 0 7 0}$ \\
& & $\mathbf{1}$ & $\mathbf{2}$ & $\mathbf{1}$ & $\mathbf{2}$ & $\mathbf{1}$ & $\mathbf{2}$ & $\mathbf{1}$ & $\mathbf{2}$ \\
\hline OTA & 29.9 & 21.5 & 22.8 & 20.8 & 23.8 & 27.8 & 31.6 & 32.1 & 30.5 \\
FP nov-abr & 14.6 & 9.2 & 8.4 & 8.4 & 8.3 & 7.9 & 7.6 & 7.2 & 6.7 \\
PRE may-oct & 11.1 & 17.1 & 15.6 & 18.8 & 14.6 & 19.3 & 23.8 & 20.1 & 25.5 \\
OT dic-feb & 8.2 & 10.3 & 12.8 & 11.8 & 12.6 & 11.2 & 13.9 & 11.5 & 14.3 \\
IH dic-feb & 7.2 & 9.8 & 8.0 & 11.1 & 9.8 & 13.7 & 8.6 & 13.9 & 11.1 \\
TM may-oct & 2.6 & 3.7 & 1.6 & 2.2 & 1.8 & 2.5 & 1.7 & 2.3 & 1.5 \\
\hline
\end{tabular}

OTA= oscilación térmica anual; $\mathrm{PRE}=$ precipitación; $\mathrm{FP}=$ fotoperiodo; $\mathrm{OT}=$ oscilación térmica; $\mathrm{IH}=$ índice de humedad; TM= temperatura mínima.

Figura 3: Variables ambientales que más influyen en la probabilidad de presencia de $B$. curtipendula
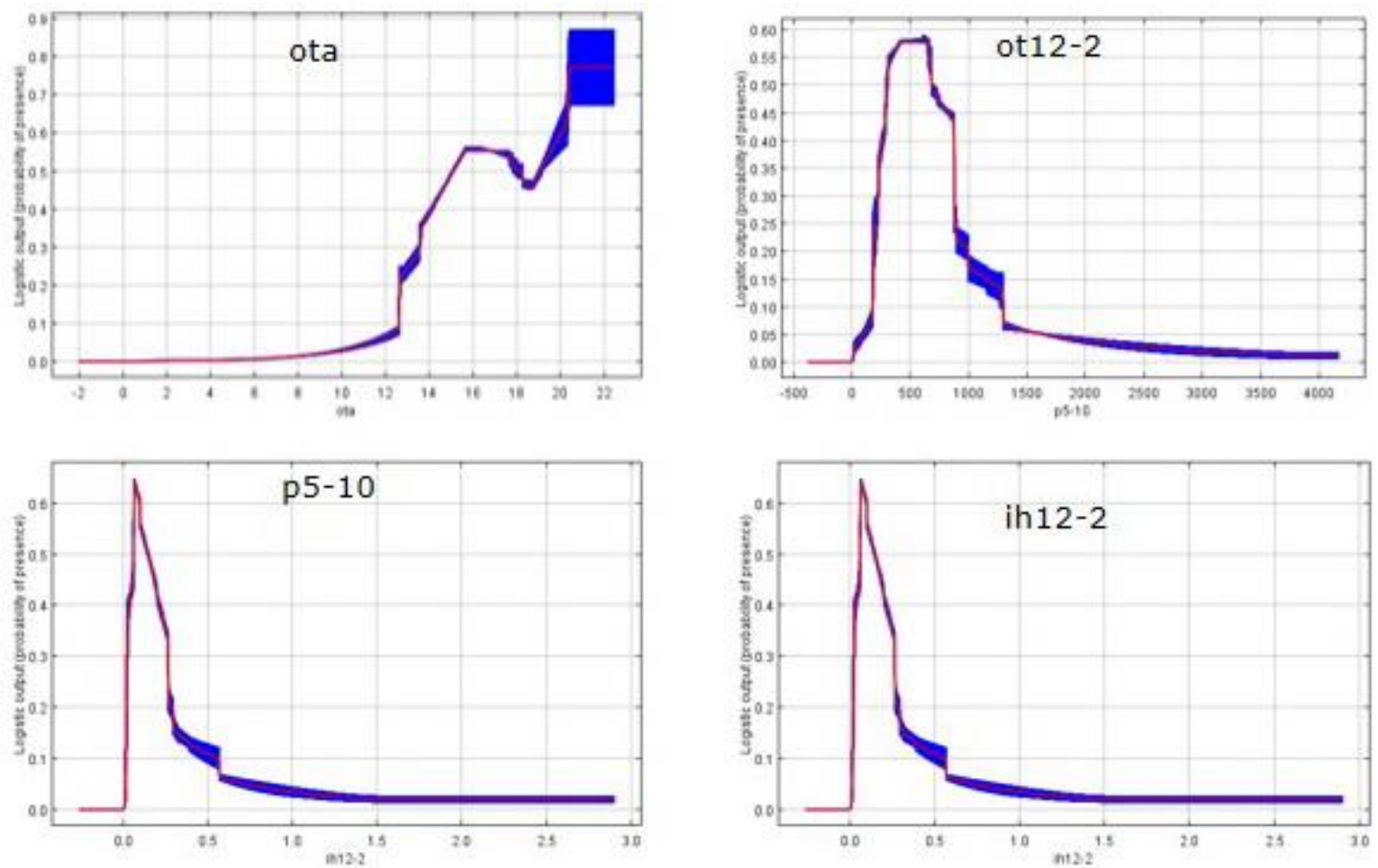

Lado izquierdo: cambios en la predicción de cada variable de la muestra promedio; lado derecho: cambios en la predicción de las variables ambientales por separado. El área sombreada representa la desviación estándar (ota, oscilación térmica anual; p5-10, precipitación may-oct; ot 12-2, oscilación térmica dic-feb; ih12-2, índice de humedad diciembre-febrero). 
La precipitación anual de manera directa no resultó uno de los principales descriptores ecológicos en la distribución de B. curtipendula, pero sí de manera indirecta mediante el índice de humedad anual y estacional, son las variables que contribuyen a explicar la presencia de la especie. Con base en la Figura 3 se puede inferir que $B$. curtipendula tiene una mayor probabilidad de estar presente en áreas con índices de humedad anual de $0.2 \mathrm{y}$ 0.5; a partir de este punto disminuye rápidamente su probabilidad a medida que este índice se acerca a 1.0; de acuerdo con estos índices de humedad, B. curtipendula se distribuye de manera natural en las zonas áridas y semiáridas ${ }^{(30,31)}$ y disminuye su presencia en las zonas subhúmedas y húmedas de México. La contribución del índice de humedad en los meses de diciembre a febrero indica que $B$. curtipendula requiere humedad para mantenerse en un su nicho ecológico, lo cual puede ser corroborado con la precipitación en el periodo diciembrefebrero, que contribuyó con el $5.4 \%$ en la distribución de la especie, incluso en mayor medida que la precipitación acumulada anual que tuvo $3.0 \%$. Al respecto, en una investigación realizada para $B$. curtipendula en el desierto Chihuahuense, la precipitación fue el factor clave para explicar la productividad primaria neta, principalmente cuando la lluvia se distribuye en pequeños pero frecuentes eventos durante el verano ${ }^{(32)}$; una situación similar se ha presentado con otras especies, como fue reportado en un estudio en África Occidental al modelar la ocurrencia de 302 especies de gramíneas, cuyo resultado fue que la precipitación es la variable que con más frecuencia influye en la distribución de las gramíneas en los pastizales $^{(11)}$; otras investigaciones también reportan que la lluvia es el factor determinante en la distribución de gramíneas en escala local y regional ${ }^{(33)}$. También se menciona que para 30 especies nativas de las Grandes Planicies de Estados Unidos el descriptor ambiental que más contribuyó en la probabilidad de ocurrencia de B. curtipendula fue la temperatura media anual $^{(26)}$, lo cual podría explicarse al tomar en cuenta la latitud que provoca temperaturas muy bajas con respecto a las áreas de distribución de esta especie en México. Al analizar la contribución de la precipitación anual por separado, la mayor probabilidad de presencia, está en los sitios de 450 a $750 \mathrm{~mm}$, con precipitaciones menores o mayores disminuyen la probabilidad de que esté presente esta especie.

Con respecto al fotoperiodo, la contribución fue de 9.0 y $14.6 \%$, en los meses de mayooctubre y noviembre-abril, respectivamente (Cuadro 3), con probabilidades de presencia mayores en rangos de 12:30 a 13:00 $\mathrm{h}$ en los meses de mayo a octubre y de 11:00 a 11:30 h en los meses de noviembre a abril (Figura 3); debe considerarse que $B$. curtipendula tienen una amplia variación genética que le permite adaptarse en ambientes diversos ${ }^{(33,34)}$, lo cual explica que esté presente en sitios tan extremos como Canadá y Argentina ${ }^{(29,35)}$. 


\section{Conclusiones e implicaciones}

Los dos modelos predicen y concuerdan que para los periodos 2050 y 2070, habrá un incremento de 2.8 a $5.0^{\circ} \mathrm{C}$ en la temperatura media anual, y una disminución de 3.1 a 14.4 $\%$ en la precipitación acumulada. El efecto de estos cambios en la superficie con aptitud ambiental para B. curtipendula tendrá ligeras consecuencias negativas a largo plazo, ya que disminuirá en el periodo 2050 para ambos RCP y para la climatología 2070 solo para el RCP4.5; pero se recuperará en el periodo 2070 con el RCP8.5 e incluso se predice un aumento promedio de $0.9 \%$ de la superficie con aptitud ambiental con respecto a la climatología de referencia. La disminución del área con aptitud ambiental se localiza principalmente en los estados de Chihuahua, Durango y Coahuila y en menor medida en pequeñas áreas del centro y sur de México; el incremento previsto en el área con aptitud ambiental se presentará principalmente en el noreste de Chihuahua y en el centro-norte de Coahuila. Las variables ambientales que contribuyeron en mayor medida a explicar la presencia de B. curtipendula en México fueron: oscilación térmica anual, precipitación en el periodo mayo a octubre, oscilación térmica en el periodo diciembre a febrero e índice de humedad de diciembre a febrero.

\section{Agradecimientos}

Se agradece al Dr. Carlos Morales Nieto por el acceso a la base de datos de las colectas que realizó; y a las autoridades del Centro Universitario de los Altos, de la Universidad de Guadalajara, por las facilidades otorgadas para realizar esta investigación.

\section{Literatura citada:}

1. SIAP-SAGARPA. Servicio de Información Agroalimentaria y Pesquera, Secretaría de Agricultura, Ganadería, Desarrollo Rural, Pesca y Alimentación, México. http://www.siap.gob.mx/. Consultado Feb 12, 2015.

2. Reeves MC, Moreno AL, Bagne KE, Running SW. Estimating climate change effects on net primary production of rangelands in the United States. Climate Change 2014;126:429-442. doi: 10.1007/s10584-014-1235-8.

3. Polley HW, Derner JD, Jackson RB, Wilsey BJ, Fay PA. Impacts of climate change drivers on $\mathrm{C} 4$ grassland productivity: scaling driver effects through the plant community. J Experim Botany 2014;65:3415-3424. doi:10.1093/jxb/eru009.

4. Durán PN, Ruíz CJA, González EDR, Ramírez OG. Impact of climate change on grasses cultivation potential of three altitudinal strata-agricultural lands of Mexico. African J Agric Res 2014;9:1396-1406. 
5. Ruíz CJA, Medina GG, Ramírez DJL, Flores LHE, Ramírez OG, Manríquez OJD, et al. Cambio climático y sus implicaciones en cinco zonas productivas de maíz en México. Rev Mex Cienc Agr 2011; Publ Esp 2:309-323.

6. Zarazúa-Villaseñor P, Ruíz-Corral JA, González-Eguiarte DR, Flores-López HE, RonParra J. Impacto del cambio climático sobre la agroclimatología del maíz en Ciénega de Chapala, Jalisco. Rev Mex de Cienc Agr 2011; Publ Esp 2:351-363.

7. Corrales LR, Morales NCR, Melgoza CA, Sierra TJS, Ortega GJA, Méndez ZG. Caracterización de variedades de pasto banderita [Bouteloua curtipendula (Michx.) Torr.] recomendadas para rehabilitación de pastizales. Rev Mex Cienc Pecu 2016;7(2):201-211.

8. Beltrán LS, García DCA, Loredo OC, Núñez QT, González ELA, García DCA, Hernández AJA, et al. Navajita Cecilia y Banderilla Diana: Pastos nativos sobresalientes para el Altiplano de San Luis Potosí. INIFAP-CIRNE-Campo Experimental San Luis, Folleto Técnico \# 33. 2007.

9. NRCS. "El Reno" sideoatas grama Bouteloua curtipendula (Michc.) Torr. USDA-Natural Resources Conservation Service: Manhattan KS, USA. 2011.

10. Morales NC, Quero CA, Le BO, Hernández GA, Pérez PJ, González MS. Caracterización de la diversidad del pasto nativo Bouteloua curtipendula Michx. Torr. mediante marcadores de AFLP. Agrociencia 2006;40:711-720.

11. Bocksberger G, Schnitzler J, Chatelain C, Daget P, Janssen T, Schmidt M, Thiombiano A, Zizka G. Climate and the distribution of grasses in West Africa. J Veget Sci 2016;27:306-317.

12. Pérez RIM, Roumet C, Cruz P, Blanchaird A, Autran P, Garnier E. Evidence for "a plant comunity economics spectrum" driven by nutrient and water limitations in a Mediterranean rangeland on Southern France. J Ecol 2012;100:1315-1327.

13. Báez S, Collins SL, Pockman WT, Johnson JE, Small EE. Effects of experimental rainfall manipulations on Chihuahuan desert grassland and shrubland comunnities. Oecol 2013;172:1117-1127. DOI 10.1007/s00442-2552-0.

14. Adler PB, Leiker J, Levine JM. Direct and indirect effects of climate change on a prairie plant community. PloS ONE 2009;4(9):e6887.

doi:10.1371/journal.pone.0006887.

15. Hijmans RJ, Cameron SE, Parra JL, Jones PG, Jarvis A. Very high resolution interpolated climate surfaces for global land areas. Internat J Climat 2005;25:1965-1978. 
16. Collins WJ, Bellouin N, Doutriaux-Boucher M, Gedney N, Halloran P, Hinton T, et al. Development and evaluation of an Earth-System model - HadGEM2. Geosci Model Dev 2011;4:1051-1075.

17. Donner LJ, Wyman BL, Hemler RS, Horowitz LW, Ming Y, Zhao M, et al. The dynamical core, physical parameterizations, and basic simulations characteristics of the atmpospheric component AM3 of the GFDL global coupled model CM3. J Climate 2011;24:3484-3519.

18. Van Vuuren BDP, Edmonds J, Kainuma M, Riahi K, Thomson A, Hibbard K, et al. The representative concentration pathways: an overview. Climatic Change 2011;109:5-31. DOI 10.1007/s10584-011-0148-z.

19. UNEP. The Emissions Gap Report. United Nations Environment Programme (UNEP), Nairobi. 2016.

20. GBIF (Global Biodiversity Information Facility). GBIF.org (11th February 2017). GBIF Ocurrence Download http://doi.org/10.15468/dl.gfgh2t.

21. Eastman JR. Idrisi Selva Manual Version 17. Worcester, Mass., USA: Clark Labs, Clark University; 2012.

22. Norris D. Model thresholds are more important than presence location type: Understanding the distribution of lowland tapir (Tapirus terrestris) in a continuous Atlantic forest of southeast Brazil. Tropical Conserv Sci 2014;7(3):529-547.

23. INEGI (Instituto Nacional de Estadística Geografía e Informática). Guía para interpretación cartográfica: Uso de suelo-vegetación Serie III. México. DF. 2009.

24. Merow C, Smith MJ, Silander JA. A practical guide to MaxEnt for modeling species distributions: what it does and why inputs and settings matter. Ecography 2013; 36:1058-1069.

25. Phillips SJ, Dudik M, Schapire RE. Maximum entropy modeling of species geographic distributions. Ecolog Model 2006;190:231-259.

26. Martinson EJ, Eddy ZB, Commerford JL. Biogeographic distributions of selected North American grassland plant species. Physical Geo 2011;32(6):583-602. Doi: 10.2747/0272-3646.32.6.583.

27. Elith J, Phillips SJ, Hastie T, Dudik M, Chee YE, Yates CJ. A. statistical explanation of MaxEnt for ecologists. Diversity Distrib 2011;17:43-57.

28. Phillips SJ, Dudik M. Modeling of species distributions with Maxent: new extensions and a comprehensive evaluation. Ecography 2008;31:161-175. 
29. Schellenberg MP, Biligetu B, McLeod GJ, Wang Z. Phenotypic variation of side-oats grama grass [Bouteloua curtipendula (Michx.) Torr.] collections from the Canadian prairie. Can J Plant Sci 2012;92:1043-1048.

30. UNEP. World Atlas of Desertification. 2nd ed. United Nations Environment Programme: Oxford University Press, England. 1997.

31. Robertson TR, Bell CW, Zak J, Tissue DT. Precipitation timing and magnitude differentially effect aboveground annual net primary productivity in three perennial species in a Chihuahuan dessert grassland. New Phytol 2009;181:230-242.

32. Edwards TJ, Smith SA. Phylogenetic analysis reveals the shady history of C4 grasses. PNAS 2010;107:2532-2537.

33. Morales NCR, Avendaño AC, Melgoza CA, Gil VKC, Quero CA, Jurado GP, et al. Caracterización morfológica y molecular de poblaciones de pasto banderita (Bouteloua curtipendula) en Chihuahua, México. Rev Mex Cienc Pecu 2016;7(4):455-469.

34. Chadwick AC. Bouteloua curtipendula. In: U.S. Department of Agriculture, Forest Service, Rocky Mountain Research Station, Fire Sciences Laboratory (Producer) 2003. http://www.fs.fed.us/database/feis/. Accesed Apr 5, 2017.

35. Siqueiros DME, Ainouche M, Columbus JT, Ainouche A. Phylogeny of the Bouteloua curtipendula complex (Poaceae: Chloridoideae) based on nuclear ribosomal and plastid DNA sequences from diploid taxa. Sistem Bot 2013;38(2):379-389. 